\title{
Pneumocephalus after posterior fossa exploration in the sitting position
}

\author{
UMA A. PANDIT, BARBARA J. MUDGE, T. S. KELLER, S. K. SAMRA. \\ P. KILARU, S. K. PANDIT AND P. J, COHEN
}

\begin{abstract}
Summary
Entrainment of air following exploration of posterior cranial fossa in the sitting position was studied in five patients. Intracranial pressure was monitored through a ventriculostomy catheter after closure of the dura. In three patients nitrous axide was added to the breathing mixture only after the baseline intracranial pressure had siabilised following closure of the dura. A marked rise in intracranial pressure was observed immediately. A rapid decrease in intracranial pressure occurred when nitrous oxide administration was stopped. Two patients were given nitrous oxide from the beginning. No change in intracranial pressure was noted.

Computerised tomogram on the first postoperative day revealed a significant amount of air in eight cases.
\end{abstract}

\section{Key words}

Surgery; neurological.

Complications; pneumocephalus.

Lecat (quoted by Jelsma \& Moore) ${ }^{1}$ reported a case of cranial 'aerocele' as early as 1741 . Only sporadic reports of gas in the cranium appeared during the next two centuries. Within the last decade, the importance of pneumocephalus has been stressed by some reviewers. ${ }^{2}$ Intracranial air is known to result from trauma, infection, tumour, congenital osseous defects or diagnostic radiological procedures. ${ }^{3}$ Development of tension pneumocephalus is a serious and life-threatening emergency. Intracranial air following posterior fossa surgery in the sitting position, has been recognised following a few case reports ${ }^{4,5}$ of post- operative tension pneumocephalus requiring reexploration. The frequency of intracranial air entrainment during posterior cranial fossa exploration is not known and not generally recognised. Standard neurosurgical anaesthesia text books ${ }^{6,7}$ do not mention pneumocephalus as a potential complication of posterior cranial fossa exploration in the sitting position.

We became interested in this problem when three consecutive patients at our institution demonstrated a considerable amount of subdural and intraventricular air on postoperative computerised tomogram (CT scan) following pos-

\footnotetext{
Uma A. Pandit, MD, Instructor of Anesthesiology, Barbara J. Mudge, MD, Assistant Professor of Anesthesiology, Ted S. Keller. MD, House Officer in Surgery, Satwant K. Samra, MD. Assistant Professor of Anesthesiology. Prasad Kilaru, MD, Instructor of Anesthesiology, Sujit K. Pandit, MD, Professor of Anesthesiology, Peter J. Cohen, MD, Professor and Chairman of Anesthesiology, Department of Anesthesiology, University of Michigan Medical Center, Ann Arbor, Michigan 48109, USA.
} 
terior fossa craniotomies in the sitting position. One of them developed tension pneumocephalus and required urgent re-exploration and decompression.

The purpose of the present study was to demonstrate the presence or absence of air in the cranium immediately and 24 hours following posterior fossa exploration in the sitting-up position in a series of patients.

\section{Methods}

The Committee for Clinical Research and Investigation Involving Human Beings of the Veterans Administration Hospital and University of Michigan Medical Center approved this study protocol. They, however, waived the informed consent requirement provided ventriculostomy was placed as a part of surgical management.

Five adult patients were studied while undergoing posterior fossa craniotomy in the sitting position. A ventriculostomy catheter was placed at the start of the operation, and pressure changes were continuously measured by a transducer after the dura was closed.

Anaesthesia was induced in all patients with appropriate doses of barbiturate and narcotics. Tracheal intubation was facilitated by the use of a non-depolarising muscle relaxant. Anaes- thesia was maintained with narcotics and a volatile anaesthetic agent, the latter was added to the anaesthetic mixture after hypocapnia was established by hyperventilation. The patients were divided into two groups according to the protocol, shown schematically in Fig. la and $1 b$.

\section{Group 1 (Fig. Ia)}

In three patients, nitrous oxide was excluded from the anaesthetic mixture until 10 minutes after the dura was closed. Two of these patients received only oxygen and one patient received nitrogen and oxygen mixture, as a carrier gas for the volatile anaesthetic agents, in addition to the narcotics. Ten minutes after the dura was closed, after obtaining the baseline intracranial pressure, $50-70 \%$ nitrous oxide in oxygen was administered. The partial pressure of arterial oxygen was maintained between 100 and 150 $\mathrm{mmHg}$. The partial pressure of arterial carbon dioxide remained constant during the pressure recordings. Nitrous oxide was administered for approximately 30 minutes while recording intracranial pressure continuously. Monitoring of ICP was maintained after discontinuation of nitrous oxide (10-20 minutes in two cases). One

(a)

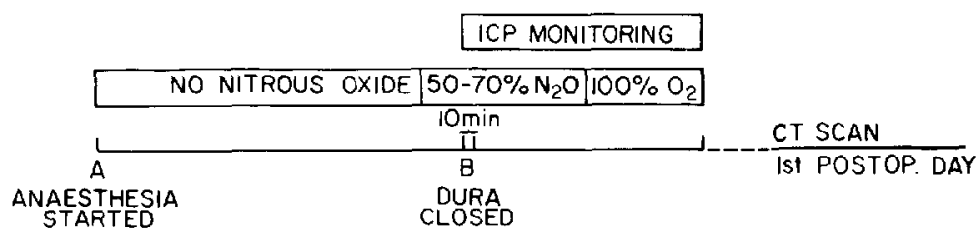

(b)

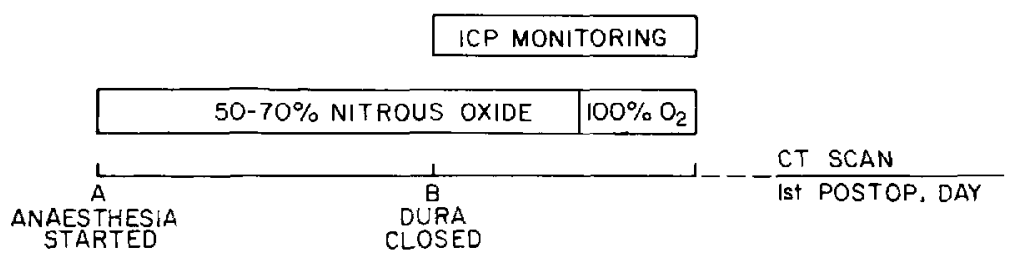

Fig. 1. Schemes of the experimental protocol. (a) Study group; (b) control group. 
patient became too light to continue with the pressure monitoring 3 minutes after nitrous oxide was stopped.

\section{Group II (Fig. 1b)}

Two cases acted as controls. They received the usual concentration $(50-70 \%)$ of nitrous oxide during the maintenance of anaesthesia. The pressure recordings were started after the dura was closed, and continued for 25 minutes. In one case, ICP records were made for 6 minutes after discontinuation of nitrous oxide. In another, the pressure recording had to be terminated because the patient started to buck on the tracheal tube.

Direct arterial pressure was recorded every minute during the ICP pressure recording. $\mathrm{PaO}_{2}$ and $\mathrm{PaCO}_{2}$ were not allowed to change significantly. No other medications (e.g. anticholinergic or cholinesterase agents) or non-surgical stimulus (e.g. movement of the head, suctioning of throat) were allowed during this time. The study was stopped if the patient started to buck or cough.

$C \Gamma$ scans of the cranium were performed in all cases on the first postoperative day to demonstrate any residual gas.

\section{Results}

Figure 2a shows the percentage changes in ICP in three cases when nitrous oxide was administered after the dura was closed. Immediately following the administration of $50-70 \%$ nitrous oxide, ICP started rising and the peak pressure was obtained in about 25 minutes. In two cases, nitrous oxide had to be discontinued because the ICP reached $20 \mathrm{mmHg}$, our predecided upper limit. The maximum changes in three cases were 240,233 and $88 \%$, respectively, compared with the baseline control. In actual numbers, they represent changes from 6 to $20,-5$ to +7 , and 11 to $20 \mathrm{mmHg}$. Figure $2 \mathrm{~b}$ shows the pressure changes in the three cases, on discontinuation of nitrous oxide when pressure reached its peak and plateaued. The graphs show percentage change compared with pre-nitrous oxide control. There was a rapid fall in pressure in one case and the ICP approached the baseline value in 7 minutes. In another case, pressure declined to one-half of its peak value in 17 minutes. The third case showed a tendency towards a fall of
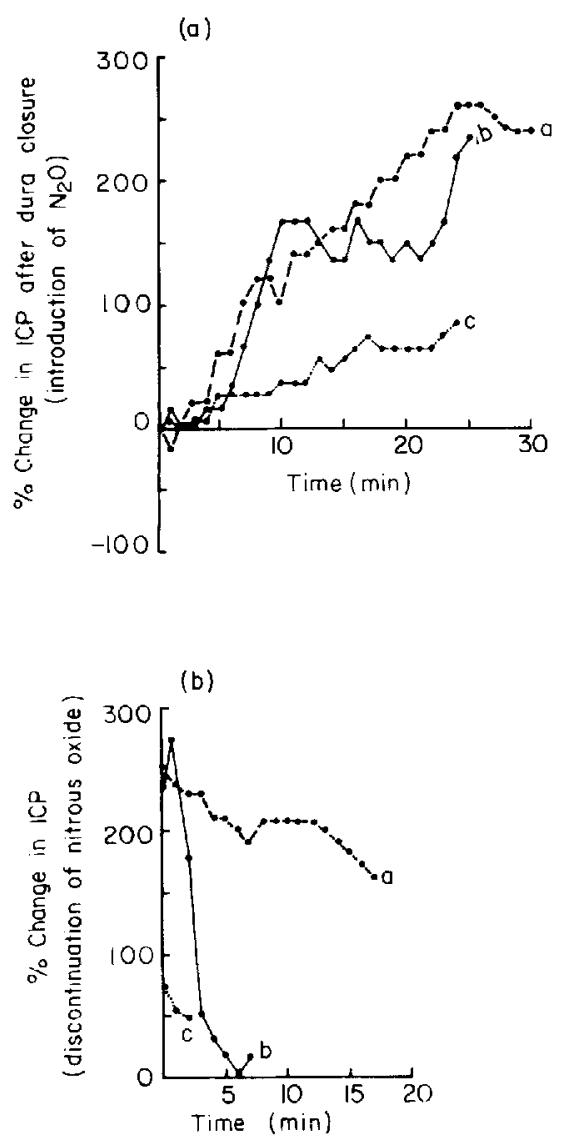

Fig. 2. Study group. (a) Ventricular pressure change (in percentage of change) 10 minutes after closure of dura. (b) Ventricular pressure changes (in percentage of change) after discontinuation of nitrous oxide.

pressure but the recordings had to be discontinued after 3 minutes because the patient started bucking on the tube.

Figure $3 \mathrm{a}$ shows the percentage pressure changes in two control cases after closure of dura, where nitrous oxide was administered throughout the anaesthetic procedure. The intracranial pressure changes upon closure of the dura were minimal in both the cases. Figure $3 b$ shows the pressure changes in one of these two cases after nitrous oxide was discontinued. The pressure fell rapidly to $30 \%$ below its starting point.

Neither arterial pressures nor pulse rate changed significantly during the study period in any patient. CT scans of all patients showed varying amounts of air in the subdural space and ventricles. The amount of air was independent of whether nitrous oxide was admin- 
(a)

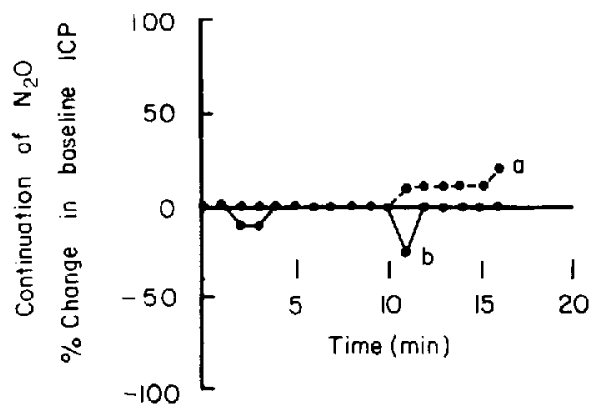

(b)

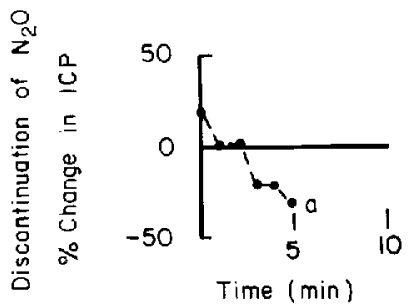

Fig. 3. Control group. (a) Ventricular pressure change (in percentage) on continuation of $\mathrm{N}_{2} \mathrm{O}$ after closure of dura. (b) Ventricular pressure changes (in percentage of change) after discontinuation of nitrous oxide.

istered throughout the anaesthetic procedure or not. Two cases in group 1 and one case in group 2 had a large amount of intracranial air in the postoperative period (Figs $4 \& 5$ ). As already mentioned in the introduction, three carlier cases showed a significant amount of intracranial air following posterior fossa exploration in sitting position. One of these required re-exploration and decompression.

\section{Discussion}

The sitting position for exploration of the posterior cranial fossa decreases intracranial pressure, decreases bleeding and provides excellent exposure of the surgical field. A major and well-known problem is air embolism. A lesser known difficulty is entrapment of air in the cranial cavity. This may be another disadvantage of this position. Dangers of entrapped air in a body cavity during anaesthesia have already been shown in cases of intestinal obstruction, pneumothorax, pneumopericardium, pneumoperitoneum, and following pneumoencephalography. ${ }^{8,9}$

Saidman \& Eger ${ }^{8}$ injected air into the cisternal space in anaesthetised dogs and then administered $75 \% \mathrm{~N}_{2} \mathrm{O}$. They demonstrated a steep rise in cisternal pressure, occurring within 10 minutes after nitrous oxide was begun. Within 10 minutes of discontinuation of $\mathrm{N}_{2} \mathrm{O}$, the cisternal pressures fell to near baseline. Eger \& Saidman ${ }^{9}$ have also demonstrated that the volume of intrapleural gas is doubled in 10 minutes and tripled in a half to three-quarters of an hour when $70-80 \% \mathrm{~N}_{2} \mathrm{O}$ was added to the inhaled mixture. There was a marked increase in volume for the first 20 minutes and then the rate of increase declined. By using a model similar to that of Saidman and Eger, we have shown a significant rise in ICP, to more than $200 \%$, when $60-70 \% \mathrm{~N}_{2} \mathrm{O}$ was added to the inhaled mixture after the dura was closed in patients not receiving this agent until this time. The rate of rise in pressure and the quick decline in ICP on discontinuation of $\mathrm{N}_{2} \mathrm{O}$ indicate the presence of air in the cranium. CT scan on all the eight patients done on the first postoperative day revealed entrapped air in varying amounts. Some of the air must have already been absorbed by this time.

The purpose of this study was to demonstrate the presence or absence of air in the cranium after dural closure following posterior cranial fossa exploration. Both administration of nitrous oxide with dural closure and CT scanning demonstrated the presence of air in all cases. The minimal change in ICP in the control group 2 patients is probably due to the fact that molecular movement of $\mathrm{N}_{2} \mathrm{O}$ into the intracranial air had already taken place. The lack of change in ICP in group 2 patients confirms that the rapid rise in ICP in group 1 patients was not due to mechanical effects of dural closure.

The ideal anaesthetic technique for posterior cranial fossa exploration remains debatable. Friedman et al. ${ }^{10}$ recently have shown that discontinuation of nitrous oxide 30 minutes before dural closure did not prevent postoperative tension pneumocephalus in one patient. This is not surprising because the entrainment of air is a mechanical effect. In the sitting position, gravity 


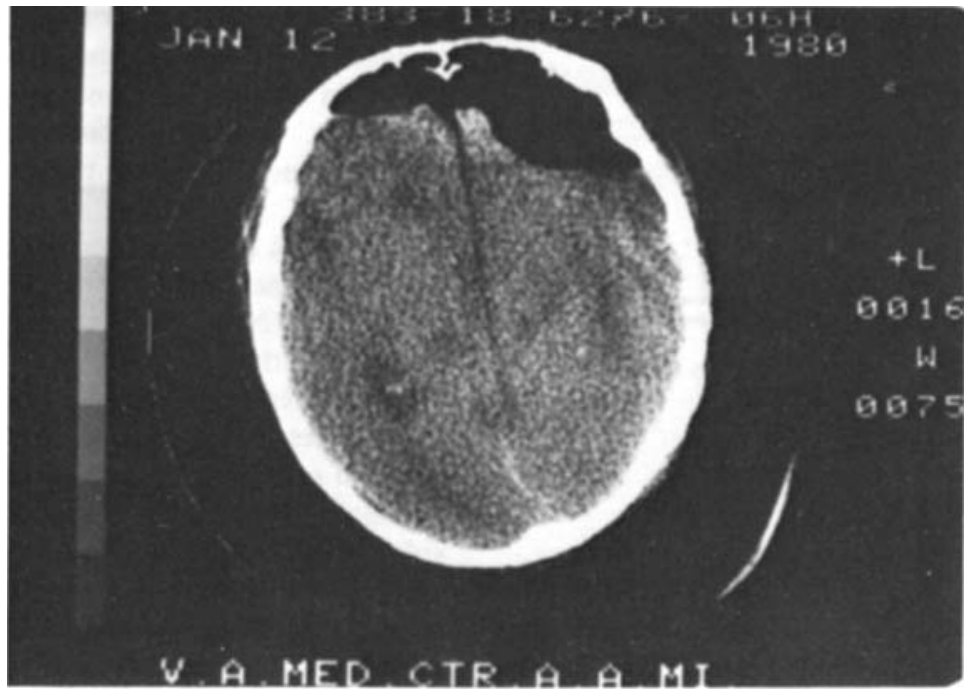

Fig. 4. CT scan 24 hours after surgery (case number 2) showing large amounts of gas in the cranium.

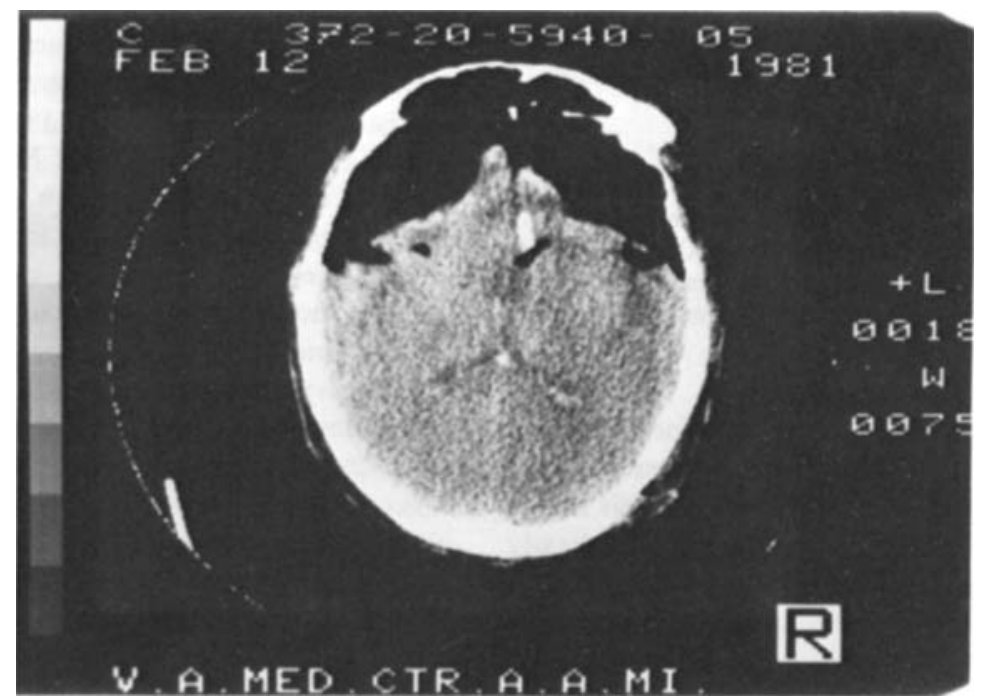

Fig. 5. CT $\operatorname{sean} 24$ hours after surgery (case number 4 ) showing large amounts of gas in the cranium.

allows the brain to settle in the cranial vault, cerebrospinal fluid (CSF) drains out, and air enters through the surgical wound to occupy the vacuum thus created at the top. This is the 'Inverted soda-pop bottle effect', described by Lunsford et al. ${ }^{4}$

It may be of value to have a ventriculostomy tube, not only to monitor ICP, but as a thera- peutic tool to release intraventricular pressure which may rise quite frequently in the postoperative period. This catheter, however, may not decompress the subdural tension in all cases, unless pulled out into the subdural space.

A slow infusion of normal saline through a subarachnoid catheter in the frontoparietal region during the surgical procedure may prevent 
the entrainment of air (Elizabeth Frost, personal communication).

\section{Conclusions}

Thus, we conclude from the results of our study and observations that air invariably enters the cranium during surgery of posterior cranial fossa in the sitting position to displace the volume of the drained CSF. This was shown by postoperative CT scans and the intracranial pressure changes after nitrous oxide administration. Anaesthetic management of these patients in the immediate postoperative period (e.g. re-exploration) should preclude $\mathrm{N}_{2} \mathrm{O}$ administration unless intracranial gas is shown to be absent on CT scan. We have observed significant amounts of air on $C T$ scans in several cases 6-7 days after posterior fossa craniotomy in the sitting position.

\section{Acknowledgments}

We especially acknowledge the secretarial assistance of Joann Duane and Cindy Hartman in preparing this manuscript.

\section{References}

1. Jelsma F, Moore DF. Cranial acrocele. American Journal of Surgery 1954; 87: 437-51.

2. NORTH JB. On the importance of intracranial air. British Journal of Surgery 1971; 58: 826-9.

3. Osborn AG, Daines JH, Wing SD, anderson RE. Intracranial air on computerized tomography. Journal of Neurosurgery 1978; 48: 355-9.

4. Lunsford LD, Makoon JC, Sheptak PE, Albin MS. Subdural tension pneumocephalus. Report of two cases. Journal of Neurosurgery 1979; 50: 5257.

5. Kitahata LM, Katz JD. Tension pneumocephalus after posterior fossa craniotomy: a complication of the sitting position. Anesthesiology 1976; 44: 448-50.

6. Cottril. JE, Turniorr H. Anesthesia and neurosurgery. St Louis: C.V. Mosby, 1980.

7. HuNTER AR. Neurosurgical anaesthesia. 2nd ed. Oxford: Blackwell Scientific, 1975.

8. SAIDMAN LJ, EgER EI. Changes in cerebrospinal fiuid pressure during pneumoencephalography under nitrous oxide anesthesia. Anesthesiology 1965; 26: 67-72.

9. Eger EI, Saidman LJ. Hazards of nitrous oxide anesthesia in bowel obstruction and pneumothorax. Anesthesiology 1965; 26: 61-5.

10. Friedman GA, Norfleet EA, Bedford RF. Discontinuance of nitrous oxide does not prevent tension pneumocephalus. Anesthesia and Analgesia 1981; 60: 57-8. 